\title{
El perfil del docente de física como factor en el desarrollo de las competencias del estudiante en el bachillerato
}

The profile of the physics teacher as a factor in the development of student competencies in high school

O perfil do professor de física como fator de desenvolvimento das competências do aluno no ensino médio

Irene Gómez Jiménez

Universidad de Guadalajara, México / Instituto Politécnico Nacional, México irene.gomez@sems.udg.mx https://orcid.org/0000-0003-1332-2159

Mario Humberto Ramírez Díaz Instituto Politécnico Nacional, México mramirezd@ipn.mx https://orcid.org/0000-0002-3459-2927

Carlos Adrián Arriaga Santos Universidad Politécnica de San Luis Potosí, México carlos.arriaga@upslp.edu.mx https://orcid.org/0000-0003-1356-406X 


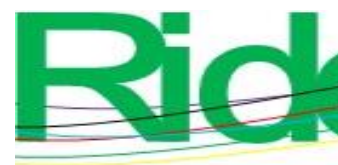

Revista Iberoamericana para la Investigación y el Desarrollo Educativo ISSN $2007-7467$

\section{Resumen}

La Escuela Preparatoria No. 7 del Sistema de Educación Media Superior (SEMS), perteneciente a la Universidad de Guadalajara (UdeG), cuenta con el modelo educativo Bachillerato General por Competencias, el cual plantea un proceso de enseñanza-aprendizaje con una orientación constructivista, que busca que el estudiante desarrolle competencias mediante la aplicación del conocimiento tomando en cuenta el contexto y la realidad social. Dentro del perfil académico del docente que imparte la unidad de aprendizaje de Física II, se menciona la necesidad de relacionar las competencias técnico-pedagógicas y la experiencia en un campo disciplinar análogo, con el propósito de realizar planeaciones didácticas, diseño, evaluación de estrategias y actividades de aprendizaje orientadas al desarrollo de competencias. Sin embargo, al terminar el bachillerato e ingresar al nivel superior, los estudiantes no obtienen notas favorables. El presente trabajo busca determinar las competencias, habilidades y conocimientos que deberá poseer un docente que enseña física para lograr que el estudiante desarrolle las competencias necesarias según el perfil de egreso en la educación media superior. Para ello, se presenta un análisis histórico de los resultados de la evaluación por competencias mediante los exámenes transversales, el perfil de los docentes (disciplinar y pedagógico), así como una evaluación docente realizada por los alumnos, una entrevista al director del plantel, a los docentes y a los estudiantes. Con ello se identifican las estrategias de actualización y capacitación para los docentes que imparten clases en la unidad de aprendizaje de Física II en la Escuela Preparatoria No. 7.

Palabras clave: física, formación docente, pedagogía, proceso de enseñanza-aprendizaje, profesor de física. 


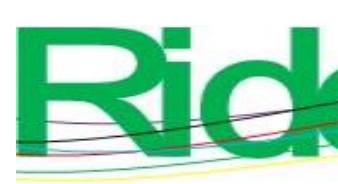

Revista Iberoamericana para la Investigación y el Desarrollo Educativo ISSN $2007-7467$

concluir o ensino médio e ingressar no nível superior, os alunos não obtêm notas favoráveis. Este trabalho visa determinar as competências, habilidades e conhecimentos que um professor que ensina física deve possuir para que o aluno desenvolva as competências necessárias de acordo com o perfil de graduação do ensino médio. Para tal, é apresentada uma análise histórica dos resultados da avaliação por competências através de exames transversais, do perfil dos docentes (disciplinar e pedagógico), bem como uma avaliação docente realizada pelos alunos, uma entrevista com o director do campus, professores e alunos. Com isso, são identificadas as estratégias de atualização e formação dos professores que lecionam na unidade de aprendizagem de Física II da Escola Preparatória no 7.

Palavras-chave: física, formação de professores, pedagogia, processo ensinoaprendizagem, professor de física.

Fecha Recepción: Mayo 2020

Fecha Aceptación: Octubre 2020

\section{Introducción}

La unidad de aprendizaje de Física se oferta dentro del Bachillerato General por Competencias del Sistema de Educación Media Superior (SEMS) que tiene la Universidad de Guadalajara (UdeG), organismo paraestatal que proporciona educación superior en el estado de Jalisco, México.

Debido a que la asignatura de Física cuenta con un mayor índice de reprobación, es necesario identificar el método a través del cual se enseña, con el fin de buscar estrategias que causen un mayor interés de los estudiantes. Sin embargo, no solo basta con identificar la estrategia más adecuada, sino que es indispensable el compromiso del docente para mejorar la enseñanza.

Es igualmente importante destacar que el perfil docente de la unidad de aprendizaje de Física II que se imparte en la Escuela Preparatoria No. 7 del SEMS de la UdeG carece de una especialización pedagógica; únicamente cuentan con un perfil profesional, esto es, una licenciatura, posgrado o diplomado en la materia.

Teniendo en cuenta lo anterior, esta investigación surge con el fin de que el perfil del docente de la asignatura en cuestión cuente con una serie de herramientas y una capacitación para que pueda generar las competencias requeridas en los alumnos de la Escuela Preparatoria No. 7. 


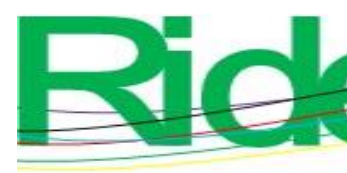

Revista Iberoamericana para la Investigación y el Desarrollo Educativo ISSN $2007-7467$

La pregunta que detonó esta investigación fue la siguiente: ¿qué competencias, habilidades y conocimientos serán convenientes que tenga un docente que enseña física para que logre que el estudiante desarrolle las competencias necesarias según el perfil de egreso en la educación media superior?

Se recurrió a una metodología cualitativa, por medio del estudio de caso, con un enfoque mixto, y una muestra cuasiexperimental. Se recabó información cualitativa mediante entrevistas con el director, los docentes y estudiantes, además de la elaboración y aplicación de un instrumento para la evaluación docente; a su vez, el promedio obtenido por los estudiantes en sus resultados transversales fue determinado y analizado. Lo anterior se complementa con la aplicación del método descriptivo, que se utiliza para evaluar el perfil docente de los profesores que imparten la unidad de aprendizaje de Física II. La técnica de investigación que se utilizó fue la entrevista por medio de preguntas generales a los docentes.

\section{Aspectos generales de la enseñanza en la unidad de aprendizaje de Física en el Bachillerato General por Competencias}

La física es un pilar básico que toda persona debe adquirir como parte de las competencias necesarias para el desarrollo en la vida diaria (Harari, 2016). Además, como es bien sabido, se encuentra íntimamente relacionada con las matemáticas. La unidad de aprendizaje de Física se oferta dentro del Bachillerato General por Competencias en los 174 planteles del SEMS de la UdeG.

Dicha asignatura cuenta con el mayor índice de alumnos no aprobados de toda la malla curricular, siguiendo con las unidades de aprendizaje de Matemáticas y Química, todas las cuales pertenecen al área de las ciencias. Esto, en muchas ocasiones, se debe a que los estudiantes no otorgan la importancia debida al tema. Automáticamente, la conciben como unidad difícil de aprobar que, a la par, carece de sentido práctico, además de que demanda mucho tiempo y hace énfasis en el pensamiento lógico-matemático y científico.

El Sistema de Análisis de Datos [SAD] (2018) de la UdeG, en específico de la Escuela Preparatoria No. 7, indica que en el calendario 2018B resultaron 916 estudiantes reprobados en general; de ello 347 no aprobaron Física I y Física II, lo cual corresponde a $37.88 \%$. Posteriormente, en el calendario 2019A, se generó un incremento: 65 alumnos más reprobaron. En ambos casos, el promedio con el que cuentan los estudiantes en esta condición 


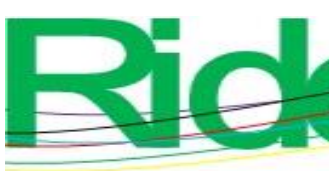

Revista Iberoamericana para la Investigación y el Desarrollo Educativo ISSN 2007 - 7467

es bajo. Y aun aquellos alumnos que logran aprobar la unidad de aprendizaje de Física, en su mayoría, no cuentan con las competencias necesarias para ingresar al nivel universitario, según los datos del proceso de admisión 2019A y 2019 B de la UdeG.

La información general que se obtuvo de los exámenes transversales de Física II de la Escuela Preparatoria No. 7, en el calendario 2019B, se encuentra vertida en la tabla 1.

Tabla 1. Promedio de resultados obtenidos por los estudiantes de Física II en el examen transversal del ciclo 2019B

\begin{tabular}{|c|c|c|c|c|c|}
\hline \multicolumn{2}{|c|}{ Academia de Física } & \multicolumn{3}{c|}{ Prepa 7 } \\
\hline $\begin{array}{c}\text { Unidad de } \\
\text { aprendizaje }\end{array}$ & $\begin{array}{c}\text { Total } \\
\text { de exámenes }\end{array}$ & Promedio & Total & Promedio & Cumplimiento \\
& & & de \\
exámenes & & \\
\hline Física II & 11533 & 37.98 & 687 & 36.4 & 82.5 \\
\hline
\end{tabular}

Fuente: Elaboración propia

En el plan de estudios del Bachillerato General por Competencias, que se divide en seis semestres, se establece que los estudiantes de primer semestre deberán cursar la unidad de aprendizaje de Física I; para el segundo semestre, se contempla que estudien Física II (Sistema de Educación Media Superior [SEMS],2015). Esta diferencia de tiempo permite que exista un rezago escolar en los primeros semestres. Cuando el estudiante pasa a tercer semestre debiendo alguna unidad de aprendizaje correspondiente a primero, solamente tendrá otra oportunidad de aprobar, por lo que es difícil, si bien no imposible, que el estudiante repruebe por más de tres veces la misma asignatura. Como señala el Reglamento General de Evaluación y Promoción de Alumnos de la Universidad de Guadalajara (H. Consejo General Universitario, 2006), el alumno tiene derecho a recuperar esta materia con el propósito de no quedar retenido durante la mitad de sus estudios.

Con el fin de buscar estrategias que despierten un mayor interés por parte de los alumnos en la materia de Física, es necesario identificar, antes que nada, el método de enseñanza aplicado actualmente. Y aunado a ello, es indispensable el compromiso del docente para mejorar la enseñanza, "ya que no se trata de convencer, ni de obligar al alumno a aprender, sino motivar en él, interés por descubrir, innovar y usar su creatividad" (Kilpatrick, Rico y Sierra, 1994, p. 137). Así pues, un factor clave en la motivación del 


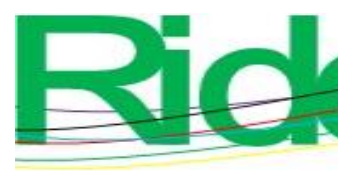

Revista Iberoamericana para la
Investigación y el Desarrollo Educativo
ISSN $2007-7467$

estudiante recae principalmente en el docente (Cermeño, 2016). Es una medida efectiva el entusiasmo, la dedicación y la orientación de las tareas que se desarrollan en el aula.

Sin duda, es necesario que los estudiantes tengan una mayor preparación para enfrentar los cambios de la vida profesional, laboral y personal. Para que esto se logre es imprescindible realizar alteraciones en los procesos de enseñanza-aprendizaje: generar en los estudiantes mayores competencias para la adquisición de conocimientos y la resolución de problemas y así lograr que sean competitivos al integrarse a la sociedad (Torres, Badillo, Valentin y Ramírez, 2014).

El estudiante no se cuestiona si el profesor tiene o no las competencias necesarias para lograr su desarrollo escolar, pues, para él, el profesor solamente imparte la asignatura; sin embargo, lo que se pretende con el presente estudio es identificar cuáles son los conocimientos, habilidades y estrategias con los que dispone para propiciar en los discentes el desarrollo de las competencias demandadas al momento de egresar. Estas características son definidas por afinidades y según las características de cada uno. El objetivo es generar una estrategia que homogenice el perfil de los docentes y favorezca el perfil de egreso de los estudiantes en la Escuela Preparatoria No. 7.

Para ello, es importante retomar información acerca de la formación docente. Según Shulman (2005), el conocimiento profesional de los docentes se distingue por siete elementos:

1) Conocimiento del contenido.

2) Conocimiento didáctico general.

3) Conocimiento del currículo.

4) Conocimiento didáctico del contenido.

5) Conocimiento de los alumnos y sus características.

6) Conocimiento de los contextos educativos.

7) Conocimiento del objetivo, las finalidades y los valores educativos y de sus fundamentos filosóficos e históricos

Un docente no debe tener simplemente conocimientos de la profesión; más allá de ser un especialista en dichos contenidos, es imprescindible que adquiera conocimientos sobre pedagogía, lo cual repercutirá al tomar en cuenta las competencias, habilidades y estrategias que se requieren para que los estudiantes desarrollen el interés por aprender y, por extensión, las competencias necesarias. 

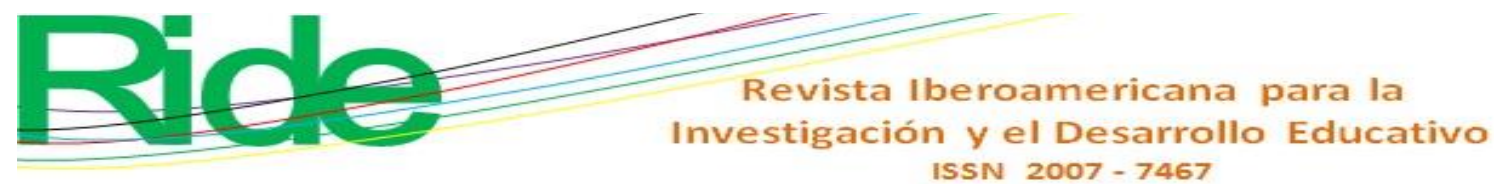

Leal (2014) habla sobre la importancia de combinar el contenido que contempla la materia con la enseñanza de esta. A esto lo llama conocimiento didáctico del contenido, y lo caracteriza a partir de cuatro elementos:

1) Está contextualizado, tanto a nivel contenido de la asignatura como a nivel instrucción.

2) Consiste en transformar, transferir y trasponer la didáctica del contenido para la enseñanza.

3) Requiere de características especiales para su formación y estudio con los profesores.

4) Para la formación de profesores, requiere reflexión y aplicación sobre la acción, la integración de psicología y contenido, la investigación de la disciplina y estudios de diferentes modos de representar el contenido a enseñar (Pinto, 2010, p.13).

Además, es importante mencionar que el perfil del docente se relaciona con una serie de conductas personales que se rescatan a partir de la diversidad de capacidades, valores, actitudes, comportamientos y estilos cognitivos del docente, lo cual es instruido al estudiante. De aquí que las características del docente admiten generar en el estudiante el interés por aprender, la inquietud del proceso de aprendizaje y la aplicación de dichos conocimientos a su vida. Araque (2017) alude a que el profesor ya no tiene que enseñar únicamente contenidos, sino que debe transmitir experiencias en el aprendizaje a fin de motivar la capacidad de asombro del estudiante. No está de más señalar que los docentes actualmente se enfrentan a más exigencias y retos por parte de los estudiantes, lo cual, de nueva cuenta, los dirige a no transmitir simplemente conocimiento, sino que se vuelve también necesario el enseñar a los estudiantes a aprender, a ser conscientes de su propio aprendizaje.

Lo anterior conlleva al cambio en el rol del docente y la relación entre docentealumno y alumno-docente. Aquí ya no exclusivamente el docente entra al aula para impartir su clase, sin llevar ninguna interacción con el estudiante, pues, aunque el docente no sea consciente, transmite valores y actitudes que generan en los alumnos la empatía para que ellos se sientan identificados con él. Molina y Pérez (2006) mencionan que los conocimientos que el docente quiere transmitir son una parte del mensaje que el alumno logra captar en el aula. 


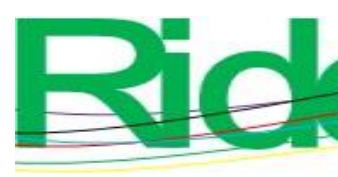

Revista Iberoamericana para la Investigación y el Desarrollo Educativo ISSN $2007-7467$

En el estudio de Covarrubias y Piñas (2004) enfatiza en la relación que existe entre el docente y sus estudiantes, la cual tiene mucho protagonismo durante el proceso de enseñanza-aprendizaje: es un factor que interviene en el aprendizaje, el comportamiento, el compromiso e incluso la actitud para el estudio.

Cuando se pretende que el estudiante aprenda en ciencia es necesario realizar procesos para implementar el desarrollo de competencias, por lo que se vuelve indispensable resolver problemas, realizar proyectos, solucionar casos, trabajar con experimentos, investigación, entre otros, con la finalidad de buscar que el alumno indague y se cuestione la manera en que resolverá cada una de estas labores para adquirir el aprendizaje, lo que lo conduce a generar su propia enseñanza por medio de un proceso natural (Frade, 2015).

¿Y por qué se enseña el desarrollo de competencias? La educación basada en competencias es creada por la necesidad de vincular la formación con la parte laboral; la escuela relaciona los conocimientos con la vida: hace práctica la teoría. Por lo que es necesario que el alumno sea capaz (al término de su formación) de demostrar la adquisición de competencias, sin dejar de lado la demostración del dominio teórico y conceptual, que son parte sustancial, en donde se evalúan los procesos y no únicamente los resultados. Cázarez y Cuevas (2007) llaman espiral de competencias a la manera como se va construyendo el aprendizaje, de forma que el andamiaje se realice con base en los resultados y productos que sirven para nuevas creaciones (aprendizaje básico).

En este sentido, Chan y Delgado (2009, citados en Torres et al., 2014) refieren que la responsabilidad del docente es "gestionar ambientes de aprendizaje, interpretar las expresiones y comportamientos del educando y comunicarse con él para apoyarlo en su proceso" (p. 137). En esa misma línea, en el acuerdo 447 de la Secretaría de Educación Pública [SEP] (2007) se establecen las características de las competencias docentes para la educación media superior, las cuales contribuyen a la formación docente y a la mejora continua del proceso de enseñanza-aprendizaje, incluyendo la de fomentar en los estudiantes la motivación durante el proceso. 


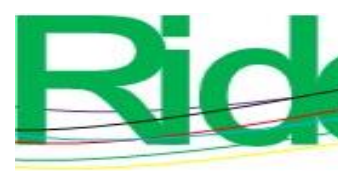

Revista Iberoamericana para la
Investigación y el Desarrollo Educativo
ISSN $2007-7467$

\section{Materiales y método}

Para la investigación del factor de competencia de los perfiles docentes de la unidad de aprendizaje de Física II del bachillerato de la UdeG, se analiza el perfil de los profesores, las competencias desarrolladas por los estudiantes y los resultados obtenidos de la evaluación docente. Dichos elementos se analizan de la siguiente manera:

- $\quad$ Con base en el conocimiento profesional, el perfil docente se analiza a partir de lo que menciona Shulman (2005).

- Las competencias adquiridas por los estudiantes se trabajan mediante los exámenes transversales que se aplican a nivel educación media superior de la UdeG, ya que se fundamentan en el programa por competencias de Física II, y muestran las competencias que el estudiante debe de adquirir al aprobar la unidad en cuestión (SEMS, 2015)

- Para la evaluación docente, se tomaron en cuenta las competencias del profesor que marca el Acuerdo 447 (SEP, 2007)

De acuerdo con Hernández, Fernández, y Baptista (2014), el enfoque utilizado para nuestro estudio de caso es mixto, con una muestra cuasiexperimental. La información cualitativa fue recabada mediante entrevistas con el director, los docentes y estudiantes, además de la elaboración, así como aplicación, del instrumento para la evaluación docente, gracias al cual se obtuvieron resultados cuantitativos, interpretados también de forma cualitativa. Por último, el promedio obtenido por los estudiantes en sus evaluaciones transversales fue revisado y evaluado (ver anexos).

La muestra se conformó a partir de los docentes que imparten la unidad de aprendizaje de Física II y los estudiantes de segundo semestre que cursan esta asignatura de la Escuela Preparatoria No. 7, la cual se encuentra ubicada en la Zona Metropolitana de Guadalajara (ZMG), en Jalisco, México. Cabe señalar que, debido a la alta demanda que tiene este plantel, se exige un alto puntaje a los estudiantes en el examen de admisión.

La escuela se encuentra conformada desde la parte académica por una planta de 196 docentes, que se distribuyen en cinco departamentos con sus respectivas academias. La de Física, junto con otras academias, forma parte del Departamento de Comprensión de la Ciencia. Dicha academia consta de 11 profesores; de ellos, solo seis son los que imparten Física II. 


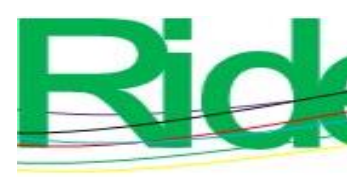

Revista Iberoamericana para la Investigación y el Desarrollo Educativo ISSN 2007 - 7467

Hasta el 2019B, la escuela registraba un total de 4989 estudiantes (distribuidos en seis semestres de 10 grupos cada uno y dos turnos). Los alumnos que cursan Física se encuentran entre el primer y segundo semestre. En Física II se cuenta con 900 estudiantes. Para dicho estudio de caso se trabajó con una muestra cuasiexperimental debido a que el estudio se realizó con tres profesores que imparten la unidad de aprendizaje de Física II y sus respectivos alumnos.

El perfil docente idóneo para la impartición de dicha unidad de aprendizaje demanda una serie de competencias técnico-pedagógicas que abarcan varios procesos: planeación didáctica, diseño y evaluación de estrategias, actividades de aprendizaje, gestión de la información y uso de tecnologías de la información y la comunicación (TIC) (SEMS, 2015).

Respecto a la experiencia en un campo disciplinar, establece una formación profesional o disciplinar en ciencias afines a la unidad de aprendizaje, preferentemente en física, geografía, ingenierías, o haber realizado cursos, diplomados u otros (presentando los documentos probatorios de instituciones reconocidas socialmente, que serán evaluados y revisados por el colegio departamental correspondiente), que avalen el conocimiento, comprensión y manejo pedagógico de los contenidos de la presente unidad de aprendizaje curricular.

Sin embargo, la mayoría de los docentes que se postulan para impartir clases de la unidad de aprendizaje Física II cuentan meramente con el perfil profesional, y no el pedagógico. Si bien los aspirantes a profesores cuentan con el grado de licenciatura, no cuentan con una formación especializada en el área pedagógica, como se puede observar en la tabla 2 .

Por lo que, durante el periodo 2012-2017, se estableció en el SEMS el Programa de Formación Docente (Profordems), que tiene como objetivo formar a los docentes de los planteles de educación media superior para contribuir al alcance del perfil docente, el cual está establecido en la Reforma Integral de Educación Media Superior (Riems), la cual, a su vez, tiene como objeto ofrecer una especialidad en competencias docentes en la Universidad Pedagógica Nacional (UPN) a las instituciones afiliadas a la Asociación Nacional de Universidades e Instituciones de Educación Superior (Anuies). Además, tiene como fin el abonar al perfil docente una serie de competencias definidas en el Acuerdo Secretaria 447 (SEP, 2007), las cuales pueden ser desarrolladas por los docentes durante el diplomado. Al concluir el curso, el docente tiene la posibilidad de acceder a un método para certificarse en 


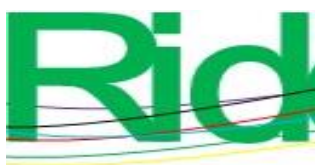

Revista Iberoamericana para la Investigación y el Desarrollo Educativo ISSN 2007 - 7467

dicha especialidad, ya sea por medio del Programa de Certificación Docente del Nivel Medio Superior (Certidems), que implica un trabajo con una aportación original e innovadora para mejorar la formación de los bachilleres, así como una entrevista para identificar los aspectos en el desarrollo de competencias docentes, o el Proceso de Evaluación de Competencias Docentes para la Educación Media Superior (Ecodems), el cual tiene dos fases, un examen de conocimiento y habilidades cognitivas y la evaluación del desempeño que recopila y verifica evidencia de desempeño, de producto y de actitud.

Los docentes que imparten Física II en la Escuela Preparatoria No. 7 cuentan con las formaciones siguientes (tabla 2):

Tabla 2. Profesores de la Preparatoria No. 7, con sus perfiles y especialidades

\begin{tabular}{|c|c|c|c|c|c|c|}
\hline & & \multicolumn{5}{|c|}{ Cantidad de profesores } \\
\hline Profesores & Licenciatura & Licenciatura & Maestría & Profordems & Ecodems & Cetidems \\
\hline Profesor A & $\begin{array}{l}\text { Ingeniero en } \\
\text { Electrónica y } \\
\text { Comunicaciones }\end{array}$ & 1 & 1 & 1 & 0 & 1 \\
\hline Profesor B & $\begin{array}{l}\text { Ingeniero } \\
\text { Mecánico } \\
\text { Eléctrico }\end{array}$ & 1 & 0 & 1 & 1 & 0 \\
\hline Profesor C & Ingeniero Civil & 1 & 0 & 1 & 1 & 0 \\
\hline Profesor D & $\begin{array}{l}\text { Ingeniero } \\
\text { Químico }\end{array}$ & 2 & 0 & 0 & 0 & 0 \\
\hline Profesor C & $\begin{array}{l}\text { Ingeniero } \\
\text { Industrial }\end{array}$ & 1 & 0 & 0 & 0 & 0 \\
\hline
\end{tabular}

Nota: El profesor A cuenta con maestría en Tecnologías para el Aprendizaje

Fuente: Elaboración propia

Con la entrevista que se hizo al director del plantel (anexo 1) se logró conseguir la información anterior. En seguida, siguiendo la tipología de Campbell y Stanley (1966), se diseñaron las tablas 3 y 4. 
Tabla 3. Simbología básica para diseños experimentales

\begin{tabular}{|l|l|}
\hline $\mathrm{R}$ & $\begin{array}{l}\text { Asignación al azar o aleatoria. Cuando aparece quiere decir que los } \\
\text { sujetos han sido asignados a un grupo de manera aleatoria. }\end{array}$ \\
\hline $\mathrm{G}$ & Grupo de sujetos o casos (G1, grupo 1; G2, grupo 2; etcétera). \\
\hline $\mathrm{X}$ & $\begin{array}{l}\text { Tratamiento, estímulo o condición experimental (presencia de algún } \\
\text { nivel o modalidad de la variable independiente). }\end{array}$ \\
\hline 0 & $\begin{array}{l}\text { Una medición de los sujetos de un grupo (prueba, cuestionario, } \\
\text { observación, etc.). }\end{array}$ \\
\hline- & $\begin{array}{l}\text { Ausencia de estímulo (nivel "cero" en la variable independiente). } \\
\text { Indica que se trata de un grupo de control o testigo. }\end{array}$ \\
\hline
\end{tabular}

Fuente: Elaboración propia

A partir de lo anterior, se diseña la caracterización docente:

1) $\mathrm{G}_{1} \mathrm{X}_{1}$ : profesor con licenciatura afín o ingeniero, cuenta con posgrado, especialidad en competencias docentes y certificación.

2) $\mathrm{G}_{2} \mathrm{X}_{2}$ : profesor con licenciatura afín o ingeniero, cuenta especialidad en competencias docentes y certificación.

3) $\mathrm{G}_{3} \mathrm{X}_{3}$ : profesor que cuenta con licenciatura afín o ingeniero, y que no tiene una especialidad en competencias docentes.

Siguiendo esta tipología, la información sería la siguiente (ver tabla 4).

Tabla 4. Tipología para trabajar la categorización de los docentes de la Preparatoria No. 7

\begin{tabular}{|l|c|c|}
\hline Profesores & Condición experimental & $\begin{array}{c}\text { Cantidad de profesores } \\
\text { en grupo }\end{array}$ \\
\hline $\mathrm{G}_{1}$ & $\mathrm{X}_{1}$ & 1 \\
\hline $\mathrm{G}_{2}$ & $\mathrm{X}_{2}$ & 2 \\
\hline $\mathrm{G}_{3}$ & $\mathrm{X}_{3}$ & 3 \\
\hline
\end{tabular}

Fuente: Elaboración propia

La entrevista docente se diseñó con preguntas generales, de opinión, para ejemplificar, de antecedentes y sensitivas, y se categorizaron con base en el conocimiento de contenido, currículo, objetivos, didáctica general, conocimiento de los alumnos y sus características, de su contexto educativo y contenido didáctico (anexo 2). 


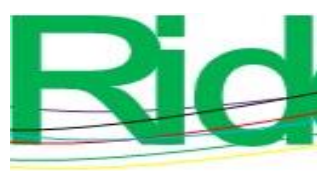

Revista Iberoamericana para la Investigación y el Desarrollo Educativo ISSN 2007 - 7467

La entrevista con los estudiantes se formuló con preguntas generales, de opinión, conocimiento, antecedentes y sensitivas. Se entrevistó a un total de 25 estudiantes por grupo de cada profesor (anexo 3).

La propuesta del instrumento de evaluación docente (anexo 4), se utilizó durante el calendario 2019A, debido a que no se contaba con ningún instrumento en el plantel; está basada en la información de competencias docentes del Acuerdo 447 (SEP, 2007). Dicho instrumento se aplicó al finalizar el semestre a una muestra de estudiantes por grupos. El objetivo era evaluar a cada profesor que impartió la unidad de aprendizaje durante ese calendario. En dicha encuesta, se realizaron preguntas sobre el quehacer docente, sus características didácticas y técnicas de enseñanza. Constó de 25 preguntas en una escala Likert. En suma, sirve para identificar los puntos de mejora en los procesos de enseñanza aprendizaje para la unidad de aprendizaje en cuestión. La tabla 5 presenta los resultados y la interpretación de la evaluación docente en cada competencia.

Tabla 5. Tabla de interpretación de la evaluación docente en cada competencia

\begin{tabular}{|c|c|c|c|c|c|c|}
\hline \multirow{2}{*}{\multicolumn{2}{|c|}{ Competencias docentes }} & \multicolumn{5}{|c|}{ Escala de interpretación } \\
\hline & & \multirow{2}{*}{$\begin{array}{c}\text { No } \\
\text { deseable }\end{array}$} & \multirow{2}{*}{$\begin{array}{c}\text { Regula } \\
\mathbf{r} \\
6-10\end{array}$} & \multirow{2}{*}{\begin{tabular}{|l} 
Bueno \\
$11-15$ \\
\end{tabular}} & \multirow{2}{*}{$\begin{array}{c}\text { Muy } \\
\text { bueno } \\
16-20\end{array}$} & \multirow{2}{*}{$\begin{array}{c}\text { Excelent } \\
\text { e } \\
21-25\end{array}$} \\
\hline 1 & Formación continua & & & & & \\
\hline 2 & Dominio de saberes & $0-3$ & $4-6$ & $7-9$ & $10-12$ & $13-15$ \\
\hline 3 & Planificación de procesos & $0-7$ & $8-14$ & $15-21$ & $22-28$ & $29-35$ \\
\hline 4 & $\begin{array}{l}\text { Aplicación de estrategias por } \\
\text { competencias }\end{array}$ & $0-8$ & $9-16$ & $17-24$ & $25-32$ & $33-40$ \\
\hline 5 & Evaluación de procesos & $0-6$ & $7-12$ & $13-18$ & $19-24$ & $25-30$ \\
\hline 6 & $\begin{array}{l}\text { Ambientes de aprendizaje } \\
\text { autónomo }\end{array}$ & $0-10$ & $11-20$ & $21-30$ & $31-40$ & $41-50$ \\
\hline 7 & $\begin{array}{l}\text { Ambientes de formación } \\
\text { integral }\end{array}$ & $0-7$ & $8-14$ & $15-21$ & $22-28$ & $29-35$ \\
\hline 8 & Participación en proyectos & 1 & 2 & 3 & 4 & 5 \\
\hline & Valoración final & $0-47$ & 48-94 & 95-141 & 142-188 & 189-235 \\
\hline
\end{tabular}

Fuente: Elaboración propia 


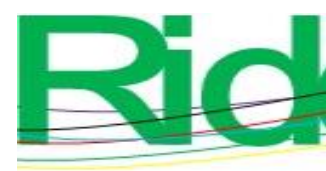

Revista Iberoamericana para la Investigación y el Desarrollo Educativo ISSN 2007 - 7467

El examen de evaluación de competencias a estudiantes, también con el nombre de transversal (anexo 5), se aplicó a todos los alumnos, de segundo hasta sexto semestre, en todas las unidades de aprendizaje; se evalúa principalmente el nivel de competencia que se desarrolla por estudiante en cada unidad. En el caso de Física II, la evaluación constó de 20 preguntas sobre temas relacionados con los contenidos allí impartidos, teniendo en cuenta las competencias genéricas puestas a continuación:

- CG5. Desarrolla innovaciones y propone soluciones a problemas a partir de métodos establecidos.

- CG5.1. Sigue instrucciones y procedimientos de manera reflexiva, comprendiendo cómo cada uno de sus pasos contribuye al alcance de un objetivo.

- CG5.2. Ordena información de acuerdo a categorías, jerarquías y relaciones.

- CG5.3. Identifica los sistemas y reglas o principios medulares que subyacen a una serie de fenómenos.

- CG5.5. Sintetiza evidencias obtenidas mediante la experimentación para producir conclusiones y formular nuevas preguntas.

Dicho examen fue validado por la Dirección de Educación Continua, Abierta y a Distancia de la UdeG, la cual se conforma por docentes de toda la red del SEMS que imparten la unidad de aprendizaje de Física, quienes aprobaron los reactivos con el reflejo de nivel de logro de competencia desarrollado por el estudiante, interpretando el nivel de logro como se muestra en la tabla 6 .

Tabla 6. Tabla de interpretación de competencias del estudiante

\begin{tabular}{|c|c|c|c|c|c|c|}
\hline \multirow{2}{*}{\multicolumn{2}{|c|}{$\begin{array}{l}\text { Competencias } \\
\text { estudiantes }\end{array}$}} & \multicolumn{5}{|c|}{ Escala de interpretación } \\
\hline & & \multirow{2}{*}{$\begin{array}{c}\text { Insuficiente } \\
1\end{array}$} & \multirow{2}{*}{$\begin{array}{c}\text { Básico } \\
2\end{array}$} & \multirow{2}{*}{$\begin{array}{c}\text { Suficiente } \\
3\end{array}$} & \multirow{2}{*}{$\begin{array}{c}\text { Avanzad } \\
\text { o } \\
4\end{array}$} & \multirow{2}{*}{$\begin{array}{c}\text { Óptimo } \\
5\end{array}$} \\
\hline 1 & CG5.1 & & & & & \\
\hline 2 & CG5.2 & 0 & - & - & - & 1 \\
\hline 3 & CG5.3 & $0-1$ & $2-3$ & $4-5$ & $6-7$ & $8-9$ \\
\hline 5 & CG5.5 & $0-1$ & $2-3$ & $4-5$ & $6-7$ & $8-9$ \\
\hline & Valoración final & $0-3$ & $4-8$ & $9-13$ & 14-18 & $19-24$ \\
\hline
\end{tabular}

Fuente: Elaboración propia 


\section{Revista Iberoamericana para la Investigación y el Desarrollo Educativo ISSN 2007 - 7467}

Lo anterior permitió generar el libro de códigos expuesto en la tabla 7.

Tabla 7. Libro de códigos para la evaluación de competencias del estudiante de Física II

\begin{tabular}{|l|c|c|c|}
\hline \multicolumn{1}{|c|}{ Variable } & Categoría & Código & Preguntas \\
\hline Estudiante & S1 & $\begin{array}{c}25 \\
\text { (estudiantes) }\end{array}$ & - \\
\hline Profesor & X1 & 1 (Profesor) & - \\
\hline $\begin{array}{l}\text { Evaluación } \\
\text { de }\end{array}$ & CG5.1 & Correcto 1 & $4,5,9,12$ y 16 \\
competencias \\
de
\end{tabular}

Fuente: Elaboración propia

\section{Resultados}

Los resultados se obtienen con base en las entrevistas al docente y se rescata la siguiente información.

Figura 1. Gráfico de la experiencia en docencia de Física II

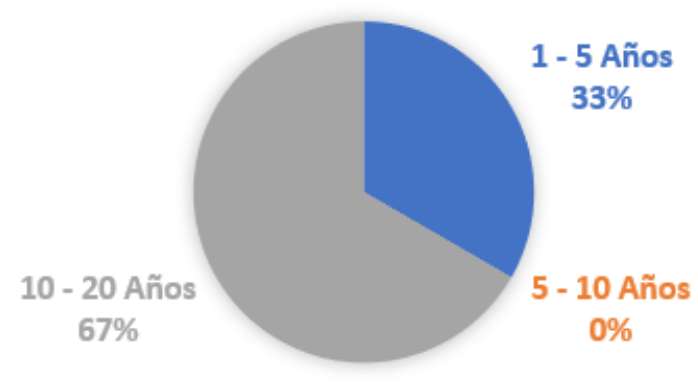

Fuente: Elaboración propia 


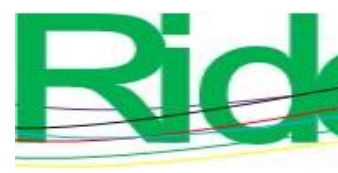

Revista Iberoamericana para la
Investigación y el Desarrollo Educativo
ISSN $2007-7467$

En la figura 1 se observa que las diferencias de edades de antigüedad en los docentes de la preparatoria son muy marcadas. Los docentes $\mathrm{G}_{1} \mathrm{X}_{1}$ y $\mathrm{G}_{2} \mathrm{X}_{2}$ tienen más de 10 años impartiendo la unidad de Física II.

Dentro de las preguntas sobre conocimiento y currículo, se obtuvo la siguiente información:

- $\quad$ Ningún docente imparte clases en la Educación Superior.

- Los docentes coinciden en que reciben ofertas de capacitación cada dos veces al año, pero procuran inscribirse a una.

- $\quad$ Los docentes consideran que por su perfil de ingeniería y antigüedad son aptos para impartir Física II.

Dentro de las preguntas de contenido didáctico general, se obtuvo la siguiente información:

- $\quad$ Los docentes coinciden en que el semestre es muy corto para los contenidos de la unidad de aprendizaje y que, de la totalidad de temas, seleccionan aquellos que podrán abarcar.

- Utilizan los criterios de evaluación marcados por la academia.

- Realizan prácticas, entre cinco y siete al semestre, sin embargo, no cuentan con los insumos correspondientes (cantidad de alumnos) ni son lo suficientemente modernos para los estudiantes.

- $\quad$ En cuanto al índice de reprobación, el docente con menos antigüedad mencionó que su índice de reprobación es medio (34 \%-67 \%), mientras que los docentes con mayor antigüedad refieren que mantienen un índice de reprobación bajo (0 \%-33 \%); además, este es un factor que se ve involucrado dependiendo el calendario escolar.

Al indagar sobre cómo ellos advierten si los estudiantes aprenden, se obtuvieron las siguientes respuestas:

- $\mathrm{G}_{1} \mathrm{X}_{1}: \mathrm{Al}$ comprender los fenómenos naturales, resolver ejercicios y aprobar el examen.

- $\mathrm{G}_{2} \mathrm{X}_{2}$ : Lo mide mediante exámenes transversales

- $\mathrm{G}_{3} \mathrm{X}_{3}$ : Lo considera cuando los alumnos entienden los puntos básicos por su retroalimentación. 

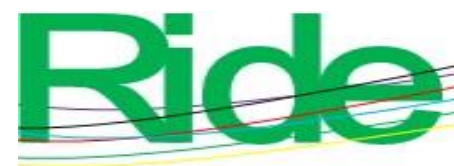

Revista Iberoamericana para la Investigación y el Desarrollo Educativo

ISSN 2007 - 7467

Sobre las acciones que realizan para lograr el aprendizaje de sus alumnos, se obtuvieron las siguientes respuestas:

- $\mathrm{G}_{1} \mathrm{X}_{1}$ : Ejemplificar cada actividad, con retroalimentación, comprender las necesidades de los alumnos.

- $\mathrm{G}_{2} \mathrm{X}_{2}$ : Elaborar preguntas y respuestas, así como elaborar ejercicios en el momento y comprobar los resultados

- $\mathrm{G}_{3} \mathrm{X}_{3}$ : Asegurando que el estudiante esté correcto dentro de los términos básicos de una clase. Ofrecer la manera más accesible de crear el conocimiento. Siempre con el pensamiento de mejorar dichas acciones para enriquecer la experiencia.

Sobre la causa por la cual sus alumnos no aprenden, se obtuvieron las siguientes respuestas:

- $\mathrm{G}_{1} \mathrm{X}_{1}$ : No ponen atención o hay falta de interés con respecto al tema.

- $\mathrm{G}_{2} \mathrm{X}_{2}$ : Por los antecedentes de los alumnos, costumbres y sobre todo los estudios previos.

- $\mathrm{G}_{3} \mathrm{X}_{3}$ : Una falta de conciencia por parte del alumno y del profesor.

Ahora bien, a partir de la evaluación realizada por los estudiantes respecto al desempeño de los docentes, se obtuvo la siguiente información con respecto a cada grupo docente dentro de la tabla 8 se encuentra el grupo $G_{1}$, en la tabla 9 se encuentra el grupo $G_{2}$ y en tabla 10 se encuentra el grupo $\mathrm{G}_{3}$ : 


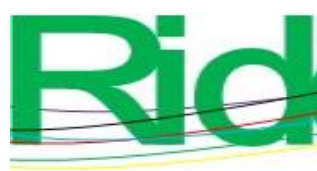

Revista Iberoamericana para la Investigación y el Desarrollo Educativo ISSN 2007 - 7467

Tabla 8. Evaluación de los docentes del grupo $\mathrm{G}_{1}$ por sus estudiantes de Física II

\begin{tabular}{|c|c|c|c|c|c|c|c|}
\hline & \multirow{2}{*}{$\begin{array}{c}\text { Competencias } \\
\text { docentes }\end{array}$} & \multicolumn{5}{|c|}{ Escala de interpretación } & \multirow{3}{*}{$\begin{array}{l}\text { Total } \\
\text { Buenc }\end{array}$} \\
\hline & & \multirow{2}{*}{$\begin{array}{c}\text { No } \\
\text { deseable } \\
0-5\end{array}$} & \multirow{2}{*}{$\begin{array}{c}\text { Regular } \\
6-10\end{array}$} & \multirow{2}{*}{\begin{tabular}{|c|} 
Bueno \\
$11-15$
\end{tabular}} & \multirow{2}{*}{$\begin{array}{c}\text { Muy } \\
\text { bueno } \\
16-20\end{array}$} & \multirow{2}{*}{$\begin{array}{c}\text { Excelente } \\
21-25\end{array}$} & \\
\hline 1 & $\begin{array}{l}\text { Formación } \\
\text { continua }\end{array}$ & & & & & & \\
\hline 2 & Dominio de saberes & $0-3$ & $4-6$ & $7-9$ & $10-12$ & $13-15$ & $\begin{array}{l}\text { Muy } \\
\text { bueno }\end{array}$ \\
\hline 3 & $\begin{array}{l}\text { Planificación de } \\
\text { procesos }\end{array}$ & $0-7$ & $8-14$ & $15-21$ & $22-28$ & $29-35$ & Bueno \\
\hline 4 & $\begin{array}{l}\text { Aplicación de } \\
\text { estrategias por } \\
\text { competencias }\end{array}$ & $0-8$ & $9-16$ & $17-24$ & $25-32$ & $33-40$ & Bueno \\
\hline 5 & $\begin{array}{l}\text { Evaluación de } \\
\text { procesos }\end{array}$ & $0-6$ & $7-12$ & $13-18$ & $19-24$ & $25-30$ & Bueno \\
\hline 6 & $\begin{array}{l}\text { Ambientes de } \\
\text { aprendizaje } \\
\text { autónomo }\end{array}$ & $0-10$ & $11-20$ & $21-30$ & $31-40$ & $41-50$ & Bueno \\
\hline 7 & $\begin{array}{l}\text { Ambientes de } \\
\text { formación integral }\end{array}$ & $0-7$ & $8-14$ & $15-21$ & $22-28$ & $29-35$ & Bueno \\
\hline 8 & $\begin{array}{l}\text { Participación en } \\
\text { proyectos }\end{array}$ & 1 & 2 & 3 & 4 & 5 & Bueno \\
\hline & Valoración final & $0-47$ & $48-94$ & $95-141$ & $142-188$ & $189-235$ & Bueno \\
\hline
\end{tabular}

Fuente: Elaboración propia 


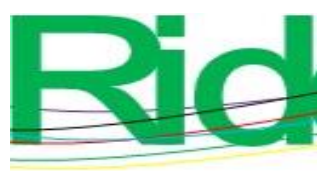

Revista Iberoamericana para la Investigación y el Desarrollo Educativo ISSN 2007 - 7467

Tabla 9. Evaluación de los docentes del grupo $\mathrm{G}_{2}$ por sus estudiantes de Física II

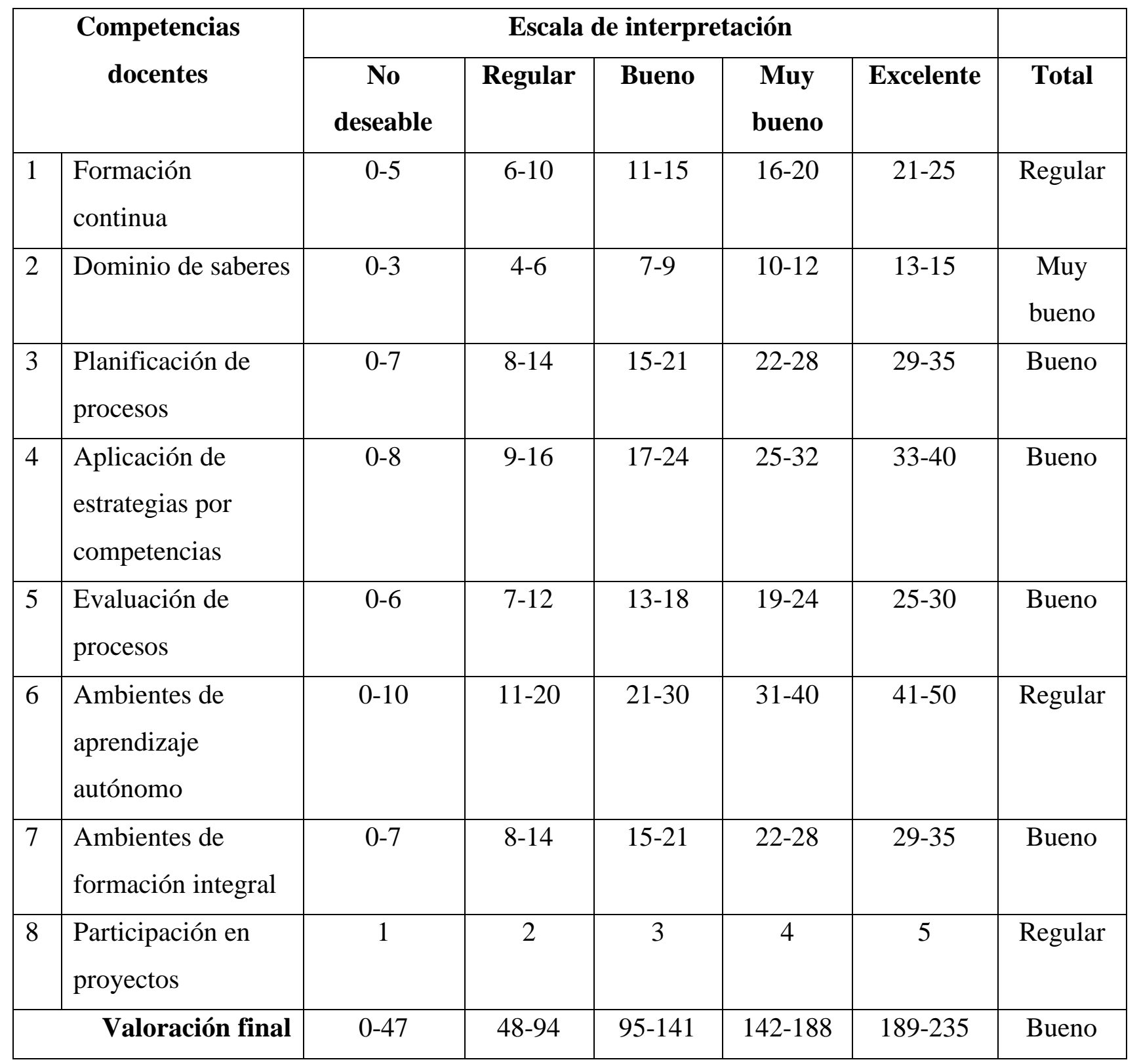

Fuente: Elaboración propia 
Tabla 10. Evaluación de los docentes del grupo $\mathrm{G}_{3}$ por sus estudiantes de Física II

\begin{tabular}{|c|c|c|c|c|c|c|c|}
\hline & \multicolumn{5}{|c|}{ Escala de interpretación } & \multirow{2}{*}{ Total } \\
\hline & Competencias aocentes & \multirow{2}{*}{$\begin{array}{c}\text { No } \\
\text { deseable }\end{array}$} & \multirow{2}{*}{$\begin{array}{c}\text { Regular } \\
6-10\end{array}$} & \multirow{2}{*}{\begin{tabular}{|l} 
Bueno \\
$11-15$
\end{tabular}} & \multirow{2}{*}{$\begin{array}{c}\begin{array}{c}\text { Muy } \\
\text { bueno }\end{array} \\
16-20\end{array}$} & \multirow{2}{*}{\begin{tabular}{|c|} 
Excelente \\
$21-25$
\end{tabular}} & \\
\hline 1 & Formación continua & & & & & & $\begin{array}{l}\text { Muy } \\
\text { bueno }\end{array}$ \\
\hline 2 & Dominio de saberes & $0-3$ & $4-6$ & $7-9$ & $10-12$ & $13-15$ & $\begin{array}{l}\text { Muy } \\
\text { bueno }\end{array}$ \\
\hline 3 & $\begin{array}{l}\text { Planificación de } \\
\text { procesos }\end{array}$ & $0-7$ & $8-14$ & $15-21$ & $22-28$ & $29-35$ & $\begin{array}{l}\text { Muy } \\
\text { bueno }\end{array}$ \\
\hline 4 & $\begin{array}{l}\text { Aplicación de } \\
\text { estrategias por } \\
\text { competencias }\end{array}$ & $0-8$ & $9-16$ & $17-24$ & $25-32$ & $33-40$ & $\begin{array}{l}\text { Muy } \\
\text { bueno }\end{array}$ \\
\hline 5 & $\begin{array}{l}\text { Evaluación de } \\
\text { procesos }\end{array}$ & $0-6$ & $7-12$ & $13-18$ & $19-24$ & $25-30$ & $\begin{array}{l}\text { Muy } \\
\text { bueno }\end{array}$ \\
\hline 6 & $\begin{array}{l}\text { Ambientes de } \\
\text { aprendizaje } \\
\text { autónomo }\end{array}$ & $0-10$ & $11-20$ & $21-30$ & $31-40$ & $41-50$ & $\begin{array}{l}\text { Muy } \\
\text { bueno }\end{array}$ \\
\hline 7 & $\begin{array}{l}\text { Ambientes de } \\
\text { formación integral }\end{array}$ & $0-7$ & $8-14$ & $15-21$ & $22-28$ & $29-35$ & $\begin{array}{l}\text { Muy } \\
\text { bueno }\end{array}$ \\
\hline 8 & $\begin{array}{l}\text { Participación en } \\
\text { proyectos }\end{array}$ & 1 & 2 & 3 & 4 & 5 & $\begin{array}{l}\text { Muy } \\
\text { bueno }\end{array}$ \\
\hline & Valoración final & $0-47$ & $48-94$ & $95-141$ & $142-188$ & $189-235$ & $\begin{array}{l}\text { Muy } \\
\text { bueno }\end{array}$ \\
\hline
\end{tabular}

Fuente: Elaboración propia

En los resultados de evaluación docente se puede identificar que, independientemente del perfil docente y si tiene o no cursos de pedagogía, los estudiantes consideran que el dominio de saberes de los tres profesores es muy bueno.

Además de observar que el profesor con menos experiencia, y que únicamente cuenta con perfil profesional, ante los estudiantes genera ambientes de formación integral y de 


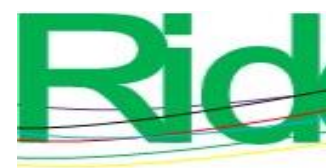

Revista Iberoamericana para la
Investigación y el Desarrollo Educativo
ISSN $2007-7467$

aprendizaje autónomo muy buenos, se apoya de instrumentos y elementos actualizados para dicho aprendizaje.

Mientras que, a partir de las preguntas sobre opinión y sensitivas hacia los estudiantes, se obtuvo la siguiente información en cada tipografía:

- $\quad 72 \%$ de los estudiantes entrevistados expresó que las estrategias que usa el profesor logran que adquieran conocimientos. El docente se asegura que presten atención, explica varias veces hasta que comprendan y quede clara la información, todo lo cual quiere decir que se toma el tiempo para aclarar dudas y logra empatizar con ellos y guiarlos en la materia $\left(\mathrm{G}_{2} \mathrm{X}_{2}\right.$ y $\left.\mathrm{G}_{3} \mathrm{X}_{3}\right)$

- $\quad 28 \%$ describe que a su profesor no le interesa si ellos entienden o no, él simplemente explica y sigue con su clase, además de poner estrictamente los ejercicios y continuar con la materia, sin tener paciencia al exponer o aclarar sus dudas $\left(\mathrm{G}_{1} \mathrm{X}_{1}\right)$.

- $\quad 10 \%$ de los estudiantes se muestra interesado por la materia y además ha buscado participar en olimpiadas de física.

- $61 \%$ de los estudiantes menciona que su profesor enlaza la unidad de aprendizaje con las actividades de la vida diaria.

- $\quad 65 \%$ menciona que en su escuela dan asesorías para los alumnos que tienen dificultad con la unidad de aprendizaje y solo $8 \%$ ha participado en las asesorías y es debido a la necesidad de pasar la asignatura.

- $\quad 37 \%$ de los estudiantes indica que su profesor algunas veces utiliza herramientas tecnológicas para su aprendizaje $\left(\mathrm{G}_{3} \mathrm{X}_{3}\right)$

Con base en los resultados de la evaluación en estudiantes de los exámenes transversales, se obtiene el promedio del desarrollo de competencias de cada profesor, según su grupo, durante los calendarios 2019A-2020A (tabla 11). 
Tabla 11. Promedio obtenido de la categorización docente de los estudiantes de Física II en los calendarios 2019A-2020A en escala de 100

\begin{tabular}{|l|c|c|c|}
\hline $\begin{array}{c}\text { Categorización } \\
\text { docente }\end{array}$ & $\begin{array}{c}\text { Promedios } \\
\text { 2019A }\end{array}$ & $\begin{array}{c}\text { Promedios } \\
\text { 2019B }\end{array}$ & $\begin{array}{c}\text { Promedios } \\
\text { 2020A }\end{array}$ \\
\hline $\mathrm{G}_{1} \mathrm{X}_{1}$ & 58.14 & 34.91 & 55.26 \\
\hline $\mathrm{G}_{2} \mathrm{X}_{2}$ & 58.59 & 37.09 & 53.63 \\
\hline $\mathrm{G}_{3} \mathrm{X}_{3}$ & 56.95 & 36.66 & 54.51 \\
\hline Promedio & 57.89 & 36.23 & 54.47 \\
\hline
\end{tabular}

Fuente: Elaboración propia

Vale la pena hacer mención que según el calendario A o B los profesores se encuentran con diferentes desafíos en las estrategias a implementar para obtener un mejor desarrollo de competencias en los estudiantes.

Los datos que se obtienen con respecto al valor en escala del desarrollo de competencias en el estudiante, de acuerdo con el profesor y el calendario, son las siguientes (ver tabla 12 y figura 2 ). 
Tabla 12. Desarrollo de competencias del grupo por calendario

\begin{tabular}{|c|c|c|c|c|}
\hline & \multicolumn{4}{|c|}{ Competencia de Estudiante } \\
\hline $2019^{a}$ & 5.1 & 5.2 & 5.3 & 5.5 \\
\hline $\mathbf{G}_{1} \mathbf{X}_{1}$ & 2.11 & 1.78 & 5.56 & 5.17 \\
\hline $\mathbf{G}_{2} \mathbf{X}_{2}$ & 2.19 & 1.83 & 5.65 & 5.15 \\
\hline \multirow[t]{2}{*}{$\mathbf{G}_{3} \mathbf{X}_{3}$} & 2.04 & 1.78 & 5.29 & 5.25 \\
\hline & \multicolumn{4}{|c|}{ Competencia de Estudiante } \\
\hline 2019B & 5.1 & 5.2 & 5.3 & 5.5 \\
\hline $\mathbf{G}_{1} \mathbf{X}_{1}$ & 1.62 & 0.47 & 3.61 & 2.82 \\
\hline $\mathbf{G}_{2} \mathbf{X}_{2}$ & 1.70 & 0.54 & 3.84 & 2.94 \\
\hline \multirow[t]{2}{*}{$\mathbf{G}_{3} \mathbf{X}_{3}$} & 1.75 & 0.57 & 3.74 & 2.99 \\
\hline & \multicolumn{4}{|c|}{ Competencia de Estudiante } \\
\hline $2020^{a}$ & 5.1 & 5.2 & 5.3 & 5.5 \\
\hline $\mathbf{G}_{1} \mathbf{X}_{1}$ & 3.17 & 1.29 & 4.88 & 5.17 \\
\hline $\mathbf{G}_{2} \mathbf{X}_{2}$ & 3.09 & 1.35 & 4.81 & 5.02 \\
\hline $\mathbf{G}_{\mathbf{3}} \mathbf{X}_{\mathbf{3}}$ & 3.07 & 1.45 & 4.87 & 4.95 \\
\hline
\end{tabular}

Fuente: Elaboración propia 

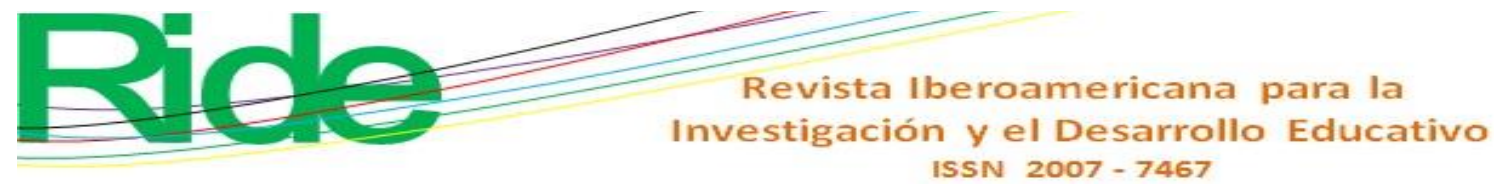

Figura 2. Promedio de grupos de cada profesor en cada calendario
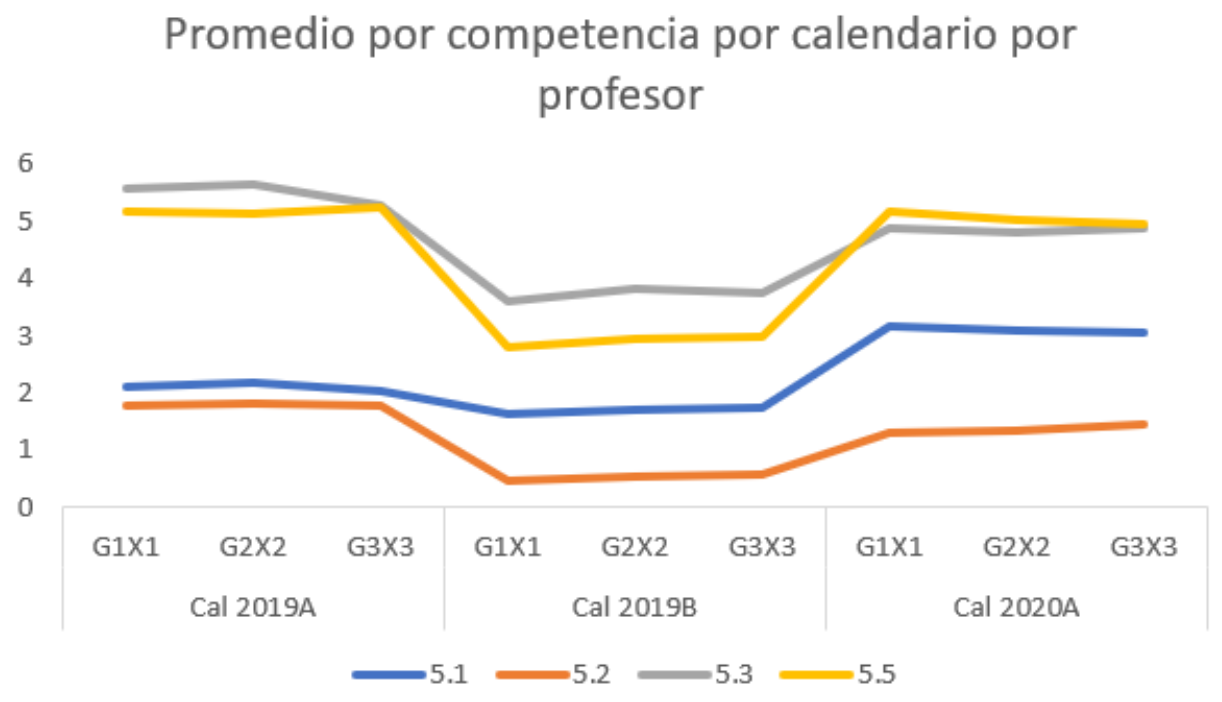

Fuente: Elaboración propia

\section{Discusión}

A partir de los hallazgos efectuados durante la investigación se puede determinar que la Escuela Preparatoria No. 7 se encuentra conformada por maestros que tienen una gran diferencia en cuanto a la experiencia impartiendo la unidad de aprendizaje de Física II. Este trabajo demuestra que hay profesores que ejercen una enseñanza tradicional para dar clases; además, se demostró que una característica particular de los docentes es su participación en la reforma integral para la educación media superior, así que comprenden la vinculación de la educación con la parte laboral, el cambio del rol del docente y las relaciones tanto dentro del aula como fuera de ella, a partir de lo cual se establecen tres asociaciones fundamentales: docente-alumno, alumno-docente y alumno-alumno; el profesor, en consecuencia, se convierte en solo un facilitador del conocimiento. Por otro lado, los maestros que tienen menos experiencia impartiendo Física II y que no cuentan con una certificación de competencias docentes generan con los estudiantes una cierta inercia que, al planificar los procesos y la aplicación de las estrategias a nivel de competencia, es evaluada como muy buena por estos últimos. 


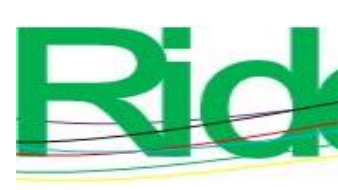

Revista Iberoamericana para la Investigación y el Desarrollo Educativo ISSN 2007 - 7467

Con respecto al conocimiento profesional, basándonos en los resultados de evaluación docente, se puede identificar que los estudiantes evalúan el dominio de saberes de los tres profesores como muy bueno, sin importar su perfil o si cuentan o no con conocimientos sobre pedagogía.

Además, el profesor con menos experiencia, que simplemente posee un perfil profesional, genera ambientes de formación integral y de aprendizaje autónomo muy buenos y se apoya en instrumentos y elementos actualizados para dicho aprendizaje.

Estos hallazgos permiten generar una relación con la pregunta de investigación desarrollada en este texto; confirman la necesidad de que el docente cuente con un perfil específico para el logro de la impartición de la enseñanza. Cabe recordar que la interrogante fue: ¿qué competencias, habilidades y conocimientos son convenientes que tenga un docente que enseña física para lograr que el estudiante desarrolle las competencias necesarias según el perfil de egreso en la educación media superior?

Principalmente, los hallazgos de este trabajo subrayan la necesidad de que el docente, además de su perfil académico (que es la selección para impartir clases), cuente con ciertas capacitaciones en temas relacionados con herramientas pedagógicas y tecnológicas para el desarrollo de las competencias de los estudiantes, participación en seminarios, foros de experiencia en el aula, la utilización de simuladores y herramientas tecnológicas. Si estas capacitaciones se realizan de manera semestral, se lograrán adquirir las competencias para que los estudiantes generen el interés por aprender, inquietud, motivación, capacidad de asombro y se enganchen por las ciencias, además de aprender cómo aplicar dichos conocimientos en su vida diaria (Arque, 2017). Se observa en los resultados que estas estrategias generan un efecto positivo en los estudiantes.

Cabe resaltar que la limitante detectada en este proceso reside en que todos los profesores de la Preparatoria No. 7 que imparten Física II son ingenieros en alguna especialidad, por lo tanto, no se puede tener una comparativa con otros perfiles y señalar, en caso de haberlas, algunas otras habilidades que logren en el estudiante el desarrollo de las competencias establecidas en el perfil de egreso. Para futuras investigaciones valdría la pena enfocarse asimismo en las condiciones en que los estudiantes ingresan a la universidad, e identificar cuál es la asimilación del conocimiento y el dominio de las habilidades y destrezas que alcanzan en la disciplina de la física, al igual que revisar la trayectoria del perfil del profesor, identificar las tareas que realiza en el aula, la articulación, la contextualización de 


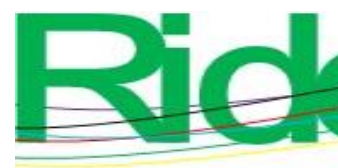

Revista Iberoamericana para la
Investigación y el Desarrollo Educativo
ISSN $2007-7467$

los contenidos que enseña con la realidad del estudiante y si motiva el aprendizaje; investigación que sería importante para complementar este trabajo.

En este sentido, se sugiere que el docente que se contrate para impartir Física II tenga una constante actualización en desarrollo, generación y aplicación de competencias. Y con ello formar ambientes de aprendizaje favorables y que influyan de manera positiva en los índices de deserción, además de lograr que los estudiantes desarrollen las competencias necesarias para su egreso de la educación media superior y para su ingreso a la vida profesional.

\section{Conclusiones}

Con respecto a la pregunta de investigación, se propone que el docente que imparte la unidad de aprendizaje de Física II tenga una constante actualización en la aplicación de competencias tanto pedagógicas como de su perfil. Esto para generar ambientes favorables de aprendizaje con el fin de que los estudiantes desarrollen las competencias necesarias una vez concluida la educación media superior.

Además, es necesario que en los calendarios B (agosto-diciembre) los docentes apliquen estrategias diferentes dentro del proceso de enseñanza-aprendizaje; más aún, no se mantengan fieles con aquellas que utilizan desde que se inicia con la experiencia docente, pues cada generación es diferente, por lo tanto, las estrategias y la manera como se enseña debe de ser también diferente.

El desarrollo de esta investigación contribuye a que en la Escuela Preparatoria No. 7 el aprendizaje se encuentre basado en la efectiva aplicación de competencias que permitan vincular la formación con la parte laboral. Para lograrlo, los docentes tienen el deber de relacionar los conocimientos con la vida cotidiana, hacer de alguna manera práctica la teoría.

Por lo que se confirma que el perfil del docente es fundamental para conseguir que el alumno sea capaz (al término de su formación) de demostrar el nivel que adquirió de competencias y manifestar un dominio teórico y práctico. El docente no debe contar simplemente con conocimientos de la profesión; es necesario que también adquiera conocimientos sobre pedagogía, lo cual repercutirá en el alumnado, al tomar en cuenta las competencias, habilidades y estrategias que cada uno requiere. 


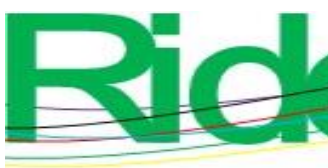

Revista Iberoamericana para la Investigación y el Desarrollo Educativo ISSN $2007-7467$

\section{Referencias}

Araque, A. (2017) El docente asombroso. (video de YouTube). TEDxUCundinamarca. Recuperado de https://www.youtube.com/watch?v=A-nw5eDP3DE.

Campbell, D. and Stanley, J. (1966). Experimental and quasi-experimental designs for research. Chicago, United States: Rand McNally \& Company.

Cázares, L. y Cuevas, J. (2007) Planeación y evaluación en competencias. Fundamentos y prácticas para el desarrollo de competencias docentes, desde preescolar hasta el posgrado. México: Trillas.

Cermeño, A. (2016) La importancia de la motivación del profesor en el aprendizaje del alumno. España: Universidad de la Rioja. Recuperado de https://biblioteca.unirioja.es/tfe_e/TFE001676.pdf.

Covarrubias, P. y Piña, R. (2004). La interacción maestro-alumno y su relación con el aprendizaje. Revista Latinoamericana de Estudios Educativos, 34(1), 47-84.

Frade, L. (2015) Desafíos a superar para desarrollar competencias en el aula. México: Patria.

Harari, Y. (2016). De animales a dioses, breve historia de la humanidad. México: Penguin Random House.

H. Consejo General Universitario. (2006). Reglamento General de Evaluación y Promoción de Alumnos de la Universidad de Guadalajara. Guadalajara, México: Universidad de Guadalajara. Recuperado de http://www.secgral.udg.mx/sites/archivos/normatividad/general/ReglamentoGralEP Alumnos.pdf.

Hernández, R., Fernández, C. y Baptista, P. (2014) Metodología de la investigación. México: McGraw-Hill. Recuperado de https://www.uca.ac.cr/wpcontent/uploads/2017/10/Investigacion.pdf.

Kilpatrick, J., Rico, L. y Sierra, M. (1994). Educación matemática e investigación. Madrid, España: Síntesis.

Leal, A. (2014) El Conocimiento Didáctico del Contenido (CDC): una herramienta que contribuye en la configuración de la identidad profesional del profesor. Magistro, 8(15), pp. 89-110. 


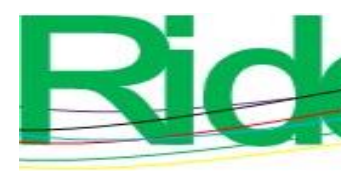

Revista Iberoamericana para la
Investigación y el Desarrollo Educativo
ISSN $2007-7467$

Molina, N. y Pérez, I. (2006). El clima de relaciones interpersonales en el aula: un caso de estudio. Paradigma, 27(2).

Pinto, J. E. (2010). Conocimiento didáctico del contenido sobre la representación de datos estadísticos: estudios de casos con profesores de estadística en carreras de psicología y educación. (tesis de doctorado). Universidad de Salamanca, Salamanca.

Secretaría de Educación Pública [SEP]. (2007) Acuerdo 447 por el que se establecen las competencias docentes para quienes impartan educación media superior en la modalidad escolarizada. México: Secretaría de Educación Pública. Recuperado de https://ransparencia.info.jalisco.gob.mx/sites/default/files/u149/ACUERDO\%2044 7.pdf.

Sistema de Análisis de Datos [SAD]. (2018). Alumnos reprobados. Escuela Preparatoria No. 7. Guadalajara, México: Universidad de Guadalajara. Recuperado de http://sad.udg.mx/cgibin/cognos.cgi?b_action=xts.run\&m=portal/cc.xts\&gohome=.

Sistema de Educación Media Superior [SEMS]. (2015) Programas de las unidades de aprendizaje. Ciencias naturales y de la salud. Guadalajara, México: Universidad de Guadalajara. Recuperado de http://www.sems.udg.mx/sites/default/files/BGC/TaesActualizadas/naturales_y_salu d_con_anexo_v03.pdf.

Shulman, L. (2005) Conocimiento y enseñanza: fundamentos de la nueva reforma. Revista de Currículum y Formación del Profesorado, 9(2), 1-30. Recuperado de https://www.ugr.es/ recfpro/rev92ART1.pdf.

Torres, A., Badillo, M., Valentin, N. y Ramírez, E. (2014) Las competencias docentes: el desafío de la educación superior. Revista Innovación Educativa. vol.14 (66) 


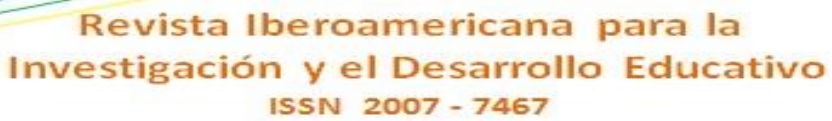

\title{
Anexo 1
}

https://www.cicata.ipn.mx/assets/files/cicata/Fisica/Documentos/Instrumentos/Preg _ent_dir_prepa_7.pdf

\begin{abstract}
Anexo 2
https://www.cicata.ipn.mx/assets/files/cicata/Fisica/Documentos/Instrumentos/Preg _ent_docentes_Fisica\%20II.pdf

\section{Anexo 3}

https://www.cicata.ipn.mx/assets/files/cicata/Fisica/Documentos/Instrumentos/Preg ent estudiantes Fisica\%20II.pdf
\end{abstract}

\begin{abstract}
Anexo 4
https://www.cicata.ipn.mx/assets/files/cicata/Fisica/Documentos/Instrumentos/Inst_ eval_estudiante_profesor.pdf

\section{Anexo 5}

https://www.cicata.ipn.mx/assets/files/cicata/Fisica/Documentos/Instrumentos/exam en_trans_comp_Fisica\%20II.pdf
\end{abstract}

